\title{
La díada éxito/fracaso escolar en las trayectorias educativas de mujeres mendocinas de sectores populares*
}

\author{
The Dyad School Success/Failure in the Academic Development of the \\ Working Class Women from Mendoza
}

Recibido 30 de enero de 2012 • Corregido 17 de mayo de 2012 • Aceptado 28 de junio de 2012

\author{
Mercedes Molina Galarza' \\ Facultad de Ciencias Políticas y Sociales \\ Universidad Nacional de Cuyo \\ Argentina \\ mmolina@mendoza-conicet.gob.ar
}

Recibido 30 de enero de 2011 • Corregido 17 de mayo de 2012 • Aceptado 28 de junio de 2012

\begin{abstract}
Resumen. La noción de rendimiento escolar da cuenta de los resultados alcanzados por los/ as estudiantes en su paso por la escuela. En su interior, encontramos dos tipos de fenómenos contrapuestos, el éxito y el fracaso escolar. La sociología de la educación ha permitido poner en cuestión ambas nociones, develando su carácter social y estrechamente vinculado con los marcos institucionales y socio-culturales en los que la escolarización se desenvuelve. El presente artículo, resultado de un trabajo de investigación desarrollado en el marco de nuestra tesis doctoral, se propone contribuir a iluminar los alcances de esa crítica, realizando un análisis de las trayectorias escolares de un grupo de mujeres mendocinas de sectores populares. Nos interesó contemplar, en primer término, las causas por las que abandonaron la escuela, aún niñas o adolescentes. Seguidamente, hemos abordado la cuestión de las oportunidades que les permitieron reingresar al sistema en la adultez, los obstáculos que debieron enfrentar y los recursos puestos en juego para poder completar el nivel medio.
\end{abstract}

Palabras claves. Rendimiento escolar, factores de abandono, oportunidades de reinserción educativa y educación de mujeres.

\footnotetext{
Licenciada en Sociología (2002) y Profesora en Sociología (2004) de la Universidad Nacional de Cuyo (Mendoza, Argentina). Doctora en Ciencias Sociales (2009) de la Facultad Latinoamericana de Ciencias Sociales (FLACSO, Buenos Aires). Fue becaria doctoral y posdoctoral del Consejo Nacional de Investigaciones Científicas y Técnicas (CONICET). Posee trabajos publicados en el campo de los estudios sociológicos sobre educación, derechos humanos, familia y género. Actualmente es Investigadora Asistente del Instituto de Ciencias Humanas, Sociales y Ambientales del CONICET, y docente de la Facultad de Ciencias Políticas y Sociales de la Universidad Nacional de Cuyo.

* El presente artículo forma parte de una investigación mayor, realizada en el marco de nuestra tesis doctoral. La misma fue financiada por medio de una beca del Consejo Nacional de Investigaciones Científicas y Técnicas (CONICET) de Argentina. Una versión abreviada del trabajo fue presentada y discutida en las I Jornadas de Estudios de Género y Feminismos: "Teorías y Políticas: desde El Segundo Sexo hasta los debates actuales" (La Plata, Argentina, 2009).
} 
URL: http://www.una.ac.cr/educare

\begin{abstract}
The notion of academic performance renders account of the results achieved by the students during their school education. Internally, there are two opposed phenomena: school success and school failure. Sociology of education has contributed to the discussion of both notions revealing their social nature closely related to the institutional and socio-cultural contexts in which education is developed. This paper is the result of a research project conducted for our doctoral thesis. Its purpose is to contribute to enlighten the scopes of this discussion, by analyzing the school development of a group of working class women from Mendoza. We were interested, in the first place, in knowing the reasons why they quit school when they were young girls or adolescents. Subsequently, we have approached the opportunities they had to access the system again during their adulthood, the obstacles they had to face and the resources to be able to complete the medium level.
\end{abstract}

Keywords. School performance, dropout causes, school reintegration opportunities, women education.

\title{
Introducción
}

El presente artículo se inscribe dentro del campo de la sociología de la educación. Nuestro propósito ha sido reconstruir las trayectorias educativas de un grupo de mujeres de sectores populares residentes en Mendoza, Argentina, que lograron en la adultez egresar de establecimientos educativos de nivel medio y obtener de este modo su título secundario. El objetivo perseguido es comprender las prácticas desarrolladas y las representaciones elaboradas en relación a las nociones de fracaso y éxito escolar, a lo largo de tales trayectorias.

Las preguntas que guiaron la indagación son: ¿qué especificidades presentan las trayectorias escolares de estas mujeres? ¿Qué circuitos educativos han recorrido durante sus infancias y adolescencias? ¿Qué factores motivaron el abandono escolar? ¿Qué oportunidades les permitieron reingresar al sistema? ¿Qué tipo de lecturas o interpretaciones del fenómeno quedan habilitadas desde una perspectiva de clase y género?

La categoría de "trayectoria educativa" es la herramienta conceptual que nos ha permitido dar cuenta, en términos diacrónicos, de los procesos de escolarización protagonizados por este grupo de mujeres, desde su ingreso al sistema educativo en la infancia, hasta su egreso del nivel secundario en la adultez. Nos interesó contemplar de manera particular los resultados escolares alcanzados en el pasado, las características de los establecimientos por los que transitaron y las causas por las que, antes de haber completado el nivel medio, abandonaron la escuela. En este sentido, la primera parte del trabajo se ha centrado en el fenómeno de lo que el sistema educativo considera como "fracaso escolar".

En la segunda parte, hemos abordado la cuestión de las oportunidades que les permitieron a las mujeres reingresar al sistema cuando ya eran adultas, los obstáculos que debieron enfrentar y los recursos puestos en juego con vistas a completar el nivel medio. Todas estas dimensiones apuntan a comprender el fenómeno del "éxito escolar" en la adultez. Como veremos, la 
imposibilidad de realizar un recorrido escolar "exitoso" en el pasado solo puede comprenderse, si se la analiza en el marco de una absoluta falta de posibilidades signada por las desigualdades sociales y de género imperantes.

Hemos trabajado con un enfoque metodológico cualitativo, orientado a lograr una comprensión profunda de los procesos socio-educativos estudiados. En términos bourdianos, la comprensión de los modos de actuar en el mundo y representar lo actuado propios de los sujetos exige poner en relación esas prácticas y representaciones (o "estrategias") con la totalidad social y temporal de la cual son producto (Bourdieu, 2000). Ello nos condujo a realizar: a) la reconstrucción de los puntos de vista de las mujeres que constituyen los sujetos de nuestra investigación; y b) la reconstrucción de las circunstancias macrosociales -determinadas referencias a la vida económica, política, legal y cultural de la sociedad argentina en general y mendocina en particular- que nos permiten situar, hallar los sentidos o descubrir las lógicas inherentes a las perspectivas de estas mujeres. Por medio de este tipo de análisis, situado e historizado, hemos procurado hacer frente al riesgo de terminar cosificando el espacio de los puntos de vista (propios de los protagonistas de la vida social) en un esfuerzo interpretativo que nada interprete, o de confundirlo con la interpretación sociológica misma (que es una operación diferente, posterior si se quiere, cuya responsabilidad recae siempre sobre el/la sociólogo/a y no debe pesar sobre los sujetos).

Las técnicas de recolección de datos han sido entrevistas en profundidad-que implicaron numerosos encuentros y largas horas de diálogo con cada una de las entrevistadas- y observaciones en terreno. El trabajo de campo se llevó a cabo durante 2006 y 2007, en barrios populares pertenecientes al aglomerado Gran Mendoza.

La unidad de análisis quedó constituida, como se ha señalado ya, por mujeres mendocinas oriundas de las clases populares, que realizaron trayectos de escolarización para personas adultas, que lograron completar el nivel medio. Se constituyó una muestra intencional, de acuerdo con criterios de dispersión geográfica (mujeres que residieran en diversos barrios de la ciudad) y dispersión etaria (mujeres pertenecientes a distintos grupos de edades). Algunas de ellas completaron el nivel secundario en establecimientos comunes para adultos/as, en tanto otras asistieron a escuelas dependientes del Plan Jefas de Hogar -un programa socio-educativo creado en Mendoza en el año 2000-. Por razones de confidencialidad, al transcribir fragmentos de las entrevistas hemos utilizado nombres ficticios, con el objeto de resguardar la identidad de las entrevistadas.

Debemos señalar que por haber trabajado con una muestra cualitativa y no representativa, los resultados obtenidos no son generalizables o extrapolables a un universo poblacional mayor. En cambio, se ha pretendido profundizar en el universo de significaciones propias de las entrevistadas, con vistas a comprender sus trayectorias escolares. Esa comprensión se orientó a dar cuenta de los procesos y las lógicas por las cuales estas personas "fracasaron" en la escuela en determinado momento de sus vidas, pero en años posteriores y en escenarios sociales apenas algo más favorables, lograron completar "exitosamente" sus estudios. 
URL: http://www.una.ac.cr/educare

\section{Algunas precisiones conceptuales}

Las profundas desigualdades sociales existentes al interior de las sociedades capitalistas hacen preciso recurrir a categorías conceptuales que den cuenta de ellas y permitan organizar lo que, de otro modo, aparecería como un entramado social denso, conflictivo e incomprensible. Por ello, hemos considerado pertinente, en esta investigación, la utilización de dos conceptos centrales para una perspectiva crítica dentro de las ciencias sociales en general y para la sociología de la educación en particular, a saber, la clase social y el género.

El primero de esos conceptos ha despertado arduos debates en las ciencias sociales, debates que han llevado a algunos autores a afirmar que las clases sociales no tienen existencia real y que, incluso, se trataría de una noción utilizada en la medida que despierta "un placer inconfesable" entre quienes hacen uso de ella (Furbank, 2005). Ante ello, nos parece necesario remarcar que, al igual que el resto de los conceptos de cualquier marco teórico operante al interior de una disciplina científica, el concepto o la "idea" de clase -como Furbank la llamaefectivamente no existe por sí misma, sino que es una herramienta conceptual construida con determinados fines heurísticos. Decir que se trata de una herramienta es afirmar que constituye un instrumento elaborado con alguna finalidad, en este caso, para facilitar el análisis y la interpretación de las relaciones y procesos sociales que tienen lugar en el interior de nuestras sociedades. Entender que los conceptos son una construcción implica aseverar que son producto del trabajo del intelecto humano, que no son ahistóricos, ni naturales, sino resultado de una cierta práctica disciplinar -la sociología, en este caso- en un momento histórico determinado. A lo anterior se suma el hecho de que el significado de cada concepto no es unívoco, sino producto de luchas y enfrentamientos sociales, así como relacional, es decir, que varía en función del marco teórico al interior del cual esa y otras categorías sean utilizadas. Dicho todo esto, nos detendremos a considerar el significado de clase social con el que hemos trabajado en nuestra investigación.

Aquellos grupos humanos que viven en condiciones de existencia similares, pertenecen a la misma clase. Una aproximación a la noción de clase indica que

Las diferencias primarias, aquellas que distinguen las grandes clases de condiciones de existencia, encuentran su principio en el volumen global del capital como conjunto de recursos y poderes efectivamente utilizables, capital económico, capital cultural, y también capital social: las diferentes clases (y fracciones de clase) se distribuyen así desde las que están mejor provistas simultáneamente de capital económico y de capital cultural hasta las que están más desprovistas en estos dos aspectos. (Bourdieu, 2006, p. 113) 
Al interior de una clase, el volumen global del capital que los diversos grupos de agentes poseen -habitualmente organizados en unidades familiares- es semejante o se encuentra dentro de ciertos márgenes; ello implica que las posiciones ocupadas por sus miembros en la estructura productiva y en la jerarquía ocupacional presentan también similitudes. La estructura del capital propia de cada grupo de agentes da cuenta de su distribución en distintas especies de recursos, como capital económico, capital cultural y capital social. Por último, el espacio social queda definido como un conjunto de relaciones entre agentes que se caracteriza por presentar tres dimensiones: el volumen del capital, su estructura y la evolución en el tiempo de estas dos propiedades.

Una segunda categoría que sirve a los fines de nuestra investigación, para comprender los actuales procesos de diferenciación social es el concepto de género. Según Scott (1990), el género es un elemento constitutivo de las relaciones sociales basadas en las distinciones entre los sexos. Esto implica que las diferencias de orden anatómico-fisiológico están acompañadas de significados social y culturalmente construidos en torno de lo que es ser mujer o varón. Las diferencias sexuales están, además, atravesadas por relaciones de poder, que le confieren a todo aquello considerado masculino una preponderancia o jerarquía superior respecto de lo considerado femenino. Existe un arsenal de doctrinas normativas en torno de estas cuestiones, que se actualizan cotidianamente en las diversas instituciones de la vida social, entre ellas, la escolar. Allí, las experiencias de habitar en un cuerpo de mujer adquieren especificidad, y dan lugar a procesos de subjetivación y socialización también específicos. Al mismo tiempo, las posibilidades de lograr el éxito en los estudios, asociadas a una serie de obstáculos y oportunidades disponibles para distintos grupos de estudiantes, son también desiguales.

En nuestra investigación, cuyo foco ha sido la educación de personas adultas, hemos trabajado con un grupo de mujeres de clases populares. Los grupos familiares a los que ellas pertenecen ocupan las posiciones más desfavorecidas de la estructura social, puesto que se ubican en la línea de pobreza ${ }^{2}$. A su tiempo, ellas se encuentran en posición de desventaja respecto de sus compañeros varones, debido a las jerarquías de género imperantes ${ }^{3}$.

La noción de rendimiento escolar da cuenta de los resultados alcanzados por los estudiantes y las estudiantes a lo largo de su paso por la escuela. En su interior, encontramos dos tipos de fenómenos contrapuestos, el éxito y el fracaso escolar. La sociología de la educación ha permitido poner en cuestión estas dos nociones -éxito y fracaso- ya que desde

\footnotetext{
Según el Instituto Nacional de Estadísticas y Censos [INDEC] (2006), en el primer semestre de 2006, el 31.4\% de la población en Argentina se encontraba en la línea de pobreza. En el aglomerado urbano Gran Mendoza, esta cifra alcanzaba al 18.3\% de la población, para el mismo período. “La medición de la pobreza con el método de la línea de pobreza' (LP) consiste en establecer, a partir de los ingresos de los hogares, si estos tienen capacidad de satisfacer -por medio de la compra de bienes y servicios- un conjunto de necesidades alimentarias y no alimentarias consideradas esenciales, [contempladas en] la canasta básica total [CBT] (INDEC, 2006, p. 10). Teniendo en cuenta la composición de cada hogar, es posible comparar el ingreso total del hogar con el valor de la CBT, para determinar si el mismo se halla o no bajo la línea de pobreza. En la presente investigación, la muestra con la que trabajamos quedó integrada por mujeres pertenecientes a hogares ubicados bajo la línea de pobreza. El valor de referencia considerado al momento de constitución de la muestra ascendía a 241,97 pesos argentinos por "adulto equivalente", en la Región Cuyo (a la cual pertenece el aglomerado Gran Mendoza), de acuerdo con el cálculo del INDEC (2006).

3 Para un análisis pormenorizado de las desventajas que enfrentan las mujeres en los más variados ámbitos de la vida social en Argentina -entre ellos, la participación en cargos públicos, el acceso a la salud, al trabajo y la educación, las relaciones familiares o la violencia de la que son víctimas- véase Equipo Latinoamericano de Justicia y Género [ELA] (2005).
} 
una perspectiva tradicional y meritocrática, aparecen como fenómenos de carácter individual y desvinculados de procesos sociales más amplios en el marco de los cuales la escolarización se desenvuelve (Cortese, Romagnoli, Bustos y Castillo, 1999). Para comprender los alcances de esa crítica, a la que pretendemos contribuir con este trabajo, es necesario definir cada uno de estos dos términos.

Desde la lógica escolar, el "éxito" implica la aprobación de los exámenes, la apropiación de la cultura escolar y la adquisición de títulos o credenciales educativas propios de cada nivel. El "fracaso" da cuenta de bajos rendimientos académicos, y de fenómenos como la sobreedad, la repitencia o el abandono (Cortese et al., 1999). La díada éxito/fracaso informa sobre los resultados escolares logrados por las estudiantes y los estudiantes en términos individuales o, en el mejor de los casos, de agregados de individuos. No obstante, invisibiliza las dimensiones sociales que permiten explicar y comprender tales resultados. Las desigualdades de clase y las jerarquías de género no son generalmente reconocidas por el discurso pedagógico ni por las políticas educativas y, en cambio, se siguen implementando mecanismos que las agudizan y perpetúan.

\section{Trayectorias escolares de las mujeres entrevistadas}

En la reconstrucción de las trayectorias escolares, comenzamos por considerar el pasaje por la escuela primaria de las mujeres que conforman la muestra. Todas ellas completaron ese nivel durante su infancia. Ello constituye un indicador de que en sus familias de origen, la escolarización básica de las niñas ha sido considerada necesaria y valiosa, y se generaron, en el plano microsocial, las condiciones para que pudieran cumplimentarla. Para contextualizar este dato proveniente de los escenarios microsociales a los que pertenecen las entrevistadas, ofrecemos un dato estadístico. En Argentina, en el año 2001, el 61\% de la población adulta del país tenía el nivel primario completo (o más años de escolaridad, inclusive), según datos del Instituto Nacional de Estadísticas y Censos [INDEC] (2001). De modo que nuestra muestra se inscribe dentro de ese $61 \%$, con al menos siete años de escolaridad completos. Sin embargo, es preciso señalar que esta población no es en absoluto homogénea en lo que hace a la calidad de la escolarización recibida, como veremos en los párrafos que siguen.

Los establecimientos a los que asistieron las mujeres entrevistadas pertenecen a un "circuito educativo" caracterizado por atender a una población estudiantil que ocupa las posiciones más bajas de la estructura social; suelen estar ubicados en zonas cercanas a sus mismos barrios de residencia; carecen, muchas veces, de la infraestructura requerida para un adecuado desenvolvimiento de la actividad académica, e imparten una educación de baja calidad.

La idea de circuitos escolares implica concebir al sistema educativo como un conjunto de instituciones y dispositivos no homogéneos ni integrados, sino profundamente fragmentados en función del origen social de quienes estudian, ofreciendo formación académica de diferente calidad y duración a los miembros de las distintas clases sociales (García-Huidobro, 1994; Romagnoli et al., 1998; Tenti, 2007). 
Al interior de un mismo circuito, las escuelas "(...) pueden ser agrupadas por sus similitudes tendencialmente estructurales (...)" (Gluz, Kantarovich y Kaplan, 2002, p. 36). Así, "(...) los sectores populares del campo y la ciudad tienden a frecuentar instituciones más pobres en términos de infraestructura, oferta curricular y recursos en general (...)" (Tenti, 2007, p. 59). La oferta escolar tiene un fuerte anclaje territorial, lo que implica que las estudiantes y los estudiantes de sectores populares solo acceden a aquellos establecimientos que se encuentran, por lo general, localizados en las zonas aledañas a donde residen. Se trata de escuelas que habitualmente no son elegidas por los miembros de las clases medias o altas, como síntoma de la fragmentación del sistema en circuitos diferenciales en función del origen de clase de los alumnos.

A continuación, nos interesa focalizar el análisis en los casos de dos entrevistadas, cuyas características son ilustrativas del tipo de escolarización primaria que han recibido las personas adultas que hoy forman parte de los sectores populares. Soledad (32 años, entrevista 31 de octubre de 2007) asistió a una escuela primaria en el Barrio Flores (ubicado en el piedemonte mendocino), en donde, según afirma, "casi por casualidad" aprendió a leer y a escribir. La define como una escuela "de muy bajo nivel", razón por la cual no la ha elegido como escuela para su hijo en el presente. La escolarización primaria no le dio a esta mujer, como a muchas otras, los capitales escolares necesarios para afrontar con éxito el nivel secundario en una escuela técnica, a la que ingresó cuando concluyó la primaria. Es sabido que este tipo de establecimientos presentan niveles de exigencia un poco más elevados que los de otras instituciones de nivel medio. Esa es la razón por la cual la joven decidió abandonar la escuela sin haber logrado pasar a segundo año, a raíz de los sucesivos "fracasos" en sus intentos de dar cumplimiento a los requerimientos escolares.

Elina, por su parte, asistió a la Escuela Hogar "Eva Perón", en donde las alumnas y los alumnos permanecen internados de lunes a viernes, y en algunos casos, se queda también los fines de semana. Esta mujer creció así alejada de su madre, y no tuvo otros familiares que se ocuparan de ella. A pesar de la privación afectiva que este modo de vida implica, la mujer aprecia la forma en que fue educada en la Escuela Hogar y considera fundamentales los valores y disposiciones que adquirió en la institución.

—AA qué edad te dejaron en la Escuela Hogar?

-Tenía yo cuatro años. [...] Sí, cuatro años más o menos. Es la edad a la que te dejan. Ahí hice la escuela, siempre la defiendo, porque me ha enseñado normas de conducta, a levantarme a las seis de la mañana, que hasta el día de hoy, en la actualidad, me levanto a la misma hora, a las seis de la mañana. Me enseñó a ser educada ante los demás, vos sabés que hay una autoridad antes que vos, esas cosas así. (Elina, 41 años. Entrevista 5 de mayo, 2006) 
URL: http://www.una.ac.cr/educare

En este fragmento se hace visible cómo, durante la segunda mitad del siglo XX, los estudiantes de origen popular aprendían en la escuela a reconocer la autoridad y las jerarquías sociales. Tal reconocimiento implicaba un fuerte disciplinamiento de quienes se esperaba que, en el futuro, siguieran ocupando las posiciones más bajas de la estructura social. Este tipo de disposiciones, inculcadas en la infancia, tienen un fuerte poder estructurante de las subjetividades, que luego se actualizará en diversos escenarios y circunstancias a lo largo de la vida. En este sentido se trata de disposiciones con un carácter duradero y transferible a aquellas nuevas situaciones que el sujeto enfrenta.

Elina terminó su instrucción primaria en la Escuela "San Marcelino Champagnat". Esta escuela depende de la congregación de los Hermanos Maristas ${ }^{4}$, perteneciente a la iglesia católica. Es una institución de gestión semi-privada, que cuenta con subsidio gubernamental y se sostiene además con fondos de la iglesia. Recibe a alumnos de sectores populares residentes en la zona de El Challao, en el piedemonte mendocino. Es conocida como "la escuelita". Según la Agencia Informativa Católica Argentina (AICA), una colaboradora de la institución hace la siguiente descripción:

Entre los cerros se asoma una escuelita con muchos alumnos paraditos en el patio. Se respira aire puro y sólo se escucha el sonido de los pájaros que pasan por ese lugar. En medio de un silencio sorprendente, los chicos se preparan para decir juntos la oración a la bandera, mientras algunos alumnos comienzan a izarla. Así, día tras día, la Escuela Champagnat, de Mendoza, recibe a 200 niños y niñas de los barrios más necesitados, para educarlos y formarlos en los valores humanos y religiosos. Esta escuelita que tiene alumnos desde $1^{\circ}$ hasta $7^{\circ}$ año es un ejemplo y está ubicada en una de las zonas más pobres del Gran Mendoza. (AICA, 2008, párr. 1)

Para analizar la idea de circuitos educativos, nos interesa detenernos en el uso del diminutivo en el fragmento anterior. El término "escuelita", diminutivo de "escuela", denota las principales características atribuidas a su población estudiantil: la pobreza y la necesidad. Se trata de alumnos también disminuidos tras el término "paraditos".

Estas denominaciones constituyen, a nuestro entender, síntomas en los que se pone de manifiesto la división de los establecimientos escolares en circuitos educativos diferenciales de la que venimos hablando, en función del origen social de los estudiantes. Otro síntoma emerge de la afirmación de que la escuela "es un ejemplo". Nos preguntamos: ¿por qué lo es? Si bien

4 De la misma congregación depende también el Colegio de los Hermanos Maristas, adonde asisten estudiantes que se encuentran en el otro extremo de la pirámide social, la elite mendocina, y goza de un reconocido prestigio. Como puede observarse, mientras "la escuelita" ha recibido el nombre de un miembro fundador de la congregación, Marcelino Champagnat, el "colegio Maristas" es identificado con el propio nombre de la congregación, lo que muestra una operación de identificación directa entre esta última y el establecimiento escolar de mayor prestigio. 
el discurso de esta mujer -a quien AICA (2008) identifica como una "colaboradora", haciendo alusión a la tarea filantrópica que realiza- no da una respuesta directa, creemos que la clave se encuentra dentro del mismo texto. Interpretamos que la institución es considerada un ejemplo porque no es esperable que, como reza el fragmento, los niños y niñas de los barrios más necesitados sean educados y formados en valores humanos y religiosos, labor que la "escuelita" sí lleva a cabo.

En el resto de las entrevistas hemos hallado que las mujeres que integran la muestra asistieron a establecimientos de similares características.

\section{Causas de abandono del nivel medio: una lectura de los fracasos escolares}

Los "fracasos" de las entrevistadas y el abandono del sistema escolar se produjeron en el nivel medio. Entre las causas de abandono, se destacan factores que dan cuenta de los lugares subordinados que ocupan las mujeres en el espacio social. En primer término, algunas de ellas han señalado la pérdida súbita de ingresos ocurrida en sus familias de origen. El factor desencadenante suele ser la ausencia, enfermedad o muerte del principal proveedor de esos hogares -el padre- dejando a las mujeres y niñas/os sumidas/os en la pobreza.

—Yo terminé séptimo grado, y me había inscripto en el Instituto Lavalle, un instituto privado.

— ¿Secundario?

-Ahá. Y yo tenía que empezar en febrero, a fines de febrero. Me había comprado los uniformes, porque era con uniforme. Y en enero, el 18 de enero, muere mi papá. Y se me truncó todo. Mi mamá no era casada con mi papá. Nos tuvo a todas nosotras, cuatro hermanas, y nunca se casó con mi papá. Mi papá trabajaba en la Municipalidad de Guaymallén, era empleado público. Pero antes estaba eso de la ley que a la concubina no le correspondía nada. No te imaginás, estuvo más de dos años para poder cobrar los seguros de mi papá, y que le dieran la pensión que le correspondía por los años trabajados ¿viste? [...] Y había que salir a trabajar, porque éramos cuatro mujeres. (Beatriz, 38 años. Entrevista 7 julio, 2006)

Quizás la problemática familiar que afectó a esta mujer no parece, a simple vista, guardar ninguna relación con las jerarquías de género imperantes. Parecería en cambio una circunstancia desgraciada, en la que el sostén del hogar perdió la vida y el resto de los miembros del mismo debieron salir a trabajar. No obstante, una mirada más atenta nos muestra que el hecho de que el varón sea el principal proveedor reposa en la una división sexual del trabajo que coloca a las 
mujeres y a los/as hijos/as en posiciones de alto riesgo, por la relación de dependencia económica que guardan con los varones. Cuando, en un momento dado, las personas dependientes deben insertarse en el mercado de trabajo, son destinadas a los puestos peor posicionados, más precarizados y peor remunerados de toda la estructura ocupacional, puestos a los que están destinados precisamente las mujeres y los niños. Ello no solo habla de una desigualdad de género, sino también de profundas inequidades generacionales.

La categoría de "vulnerabilidad" ilustra claramente este tipo de escenarios. Permite analizar de modo concomitante las amenazas y los riesgos a los que están expuestos los hogares (por ejemplo, la pérdida del ingreso) y las oportunidades o capacidades de adaptación que presentan (Moser, 1996). Hogares más vulnerables son aquellos en los que la capacidad de desplegar estrategias de adaptación o sobrevivencia ante una situación crítica es limitada, por lo que sus miembros se ven sometidos a privaciones o sacrificios considerables para sobrevivir. Tal es el caso de aquellas entrevistadas que, como Beatriz, debieron renunciar a educarse para salir a trabajar siendo aún niñas o adolescentes.

En todos los casos, los empleos a los que accedieron eran de un elevadísimo nivel de precariedad y muy bajos ingresos (venta ambulante y servicio doméstico). Así, el acceso a la actividad remunerada ha implicado para ellas una pérdida de derechos, que nada tiene que ver con la conquista del espacio público o el disfrute de ventajas asociadas al abandono de la esfera doméstica.

El segundo de los factores sindicados como causantes del abandono escolar se relaciona con la noción de inteligencia. Algunas entrevistadas han señalado que su rendimiento en la escuela era muy bajo porque no tenían capacidad intelectual suficiente para estudiar.

-No es porque no hubiera tenido los recursos, sino porque yo no quise. Yo dije que no me daba y bueno, mi papá me dijo: “¿No querés estudiar? Bueno, no estudiés, andá a trabajar."

— ¿Hasta qué año habías llegado?

- Hasta primer año nomás. Y de ahí bueno, nunca más... Porque yo siempre pensé que no me sentía capacitada para seguir estudiando. Decía yo que era burra, que me costaba estudiar, todo eso. (Carolina, 30 años. Entrevista 19 de junio, 2006)

Los modos más difundidos de concebir a la inteligencia se asientan en una idea biologicista o esencialista que daría cuenta de una cierta capacidad (o incapacidad) de los sujetos para aprender, que sería innata e inmodificable (Gardner, Kornhaber y Wake, 2005). Lo que constituye una cuestión social vinculada a los modos de aprender y enseñar, los contenidos, las finalidades de la educación y las/los destinatarias/os, es invisibilizado y confundido con un asunto psicológico o mental de carácter individual. 
Desde la perspectiva que aquí se sostiene, todas las personas estamos capacitadas para aprender cosas nuevas a lo largo de nuestra vida. Lo que sucede es que para incorporar nuevos conocimientos, se necesitan ciertos recursos o capitales intelectuales, es decir, ciertos saberes previos. Esos recursos no son innatos, no forman parte de una esencia de la persona, son en cambio "heredados" (en términos bourdianos) o adquiridos en el transcurso de la vida social. Se pone de manifiesto, entonces, la necesidad de una educación previa de calidad. En ese proceso, el origen de clase y el género tienen un peso fundamental.

Respecto del contexto familiar, la literatura sociológica ha alertado acerca de la correlación existente entre el nivel educativo alcanzado por los miembros mayores de la familia, especialmente el padre y la madre, y el éxito o fracaso escolar de los estudiantes. Se ha destacado, además, la dificultad de desarrollar una expectativa de escolarización exitosa cuando en el grupo familiar los niveles educativos alcanzados no son elevados, puesto que el "clima educativo" reinante es poco propicio (Tenti, 2007, p. 58).

Ambas cuestiones -el no haber contado con una formación previa de calidad y la falta de experiencias escolares exitosas en el núcleo familiar inmediato- son factores que contribuyen a explicar la falta de confianza en relación con las propias capacidades intelectuales por parte de las mujeres entrevistadas, lo que a su vez explica el abandono temprano de los estudios en el nivel medio.

No obstante, se debe tomar la precaución de no caer en determinismos que impidan visualizar las fisuras u oportunidades sobre las cuales las estrategias de estas mujeres se han deplegado en la adultez. Por ejemplo, en el caso de Carolina, más allá de las restricciones propias del contexto social en que ella se insertaba, hubo factores que la estimularon a reencontrarse con las propias potencialidades, de manera que logró terminar la escuela secundaria. En apartados posteriores veremos cómo un conjunto de modificaciones en el nivel institucional -al interior del sistema educativo para adultos/as- permitió que muchas mujeres mendocinas lograran completar el nivel medio, en el marco de un programa social-educativo para mujeres jefas de hogar.

Volviendo a la cuestión de los factores que explican el abandono escolar, debe destacarse que las desigualdades culturales y sociales -que se construyen a partir de ocupar posiciones de diferente jerarquía dentro de la sociedad- son traducidas como diferencias naturales entre varones y mujeres, que quedan legitimadas a la luz de una supuesta desigual distribución de dones o capacidades intelectuales. Las niñas son consideradas, en ocasiones, poco inteligentes, pero más dóciles, aplicadas y responsables para el trabajo escolar. Existe el supuesto de que poseen mayor emotividad (que los varones) y escasas capacidades para razonar en términos lógicos, lo que con el paso del tiempo va configurando ciertas trayectorias, y obstruyendo otras.

El tercer factor señalado en las entrevistas como causa del abandono escolar es el embarazo. La idea de que la escuela no es un lugar para una chica embarazada se 
corresponde con el hecho de que, al tratarse de una sociedad patriarcal, la mayoría de las políticas educativas no contemplaba el fenómeno del embarazo mientras estas mujeres eran adolescentes, con lo que se generaron escenarios altamente excluyentes. El embarazo ha estado asociado con uniones conyugales tempranas, abandono del mercado de trabajo y asunción de múltiples responsabilidades en la esfera doméstica por parte de las entrevistadas.

Intentando sostener una perspectiva que supere una lectura lineal de las entrevistas, el embarazo no puede ser considerado como el único factor interviniente por el cual el abandono escolar tuvo lugar. Se trata, en cambio, de un elemento desencadenante que se inscribe en un contexto institucional y social determinado. Así, el abandono se produjo en un escenario escolar atravesado por sentidos y expectativas respecto de lo que debe ser y hacer una adolescente estudiante de nivel medio. La maternidad adolescente no suele ser considerada esperable ni deseable, en tanto se concibe a la adolescencia como período "de moratoria" en el cual corresponde que las jóvenes se formen para las obligaciones que se asumirán en una futura adultez. Este modo de concebir la adolescencia es universalista y etnocéntrico según Fainsod (2006, p. 41), porque se basa en el modo de vivir una cierta etapa vital que corresponde a los miembros de las clases medias o altas, y supone que los miembros de los sectores populares deben inscribirse en experiencias similares. En tal sentido, hay una cierta normatividad subyacente respecto de lo que se debe ser y hacer cuando se es adolescente, y lo que escapa a esos parámetros socialmente impuestos es considerado "inapropiado". Así, la idea de lo inapropiado (como inadecuado, inoportuno o inconveniente) conlleva una manera de ejercer la violencia simbólica y produce efectos sobre las subjetividades.

La temporalidad que según el sentido común se atribuye a la adolescencia está asentada en el futuro. Los miembros de las distintas clases sociales tienen experiencias diferenciales respecto del paso del tiempo y de las dimensiones del presente y del futuro. Las dificultades de anticipar y proyectar el futuro explican que las experiencias de los sectores populares estén centradas en el presente -lo que Fainsod llama la "lógica del instante", por oposición a la "lógica de la anticipación" propia de las clases favorecidas (Fainsod, 2006, p. 57)-. La adolescencia como moratoria no puede tener para sujetos de origen popular, entonces, el mismo sentido que para los de otro origen social. No obstante, en las escuelas se actualiza este sentido único de lo que es ser adolescente, y se sostienen discursos culpabilizadores respecto de eventos que "suspenden" la etapa de moratoria, entre ellos los embarazos.

Como hemos señalado, la concepción de que la escuela no es un lugar para una chica embarazada es muchas veces un factor que contribuye al abandono escolar. Esta concepción se corresponde con el hecho de que la mayoría de las políticas escolares no están diseñadas para poblaciones de adolescentes embarazadas. Por ejemplo, hasta la sanción de la Ley № 25.273 (2000) en Argentina, las inasistencias por causa del embarazo no necesariamente se justificaban. Esta norma instituye un régimen especial de inasistencias justificadas por razones de gravidez 
para alumnas que cursen los ciclos de enseñanza básica, polimodal ${ }^{5}$ o superior no universitaria. Establece además el derecho a una hora diaria de lactancia por un período de seis meses. Antes de la entrada en vigencia de dicha ley, la posibilidad de que se contemplaran este tipo de circunstancias vinculadas al embarazo y la maternidad dependía de la buena voluntad de las autoridades del establecimiento.

La Ley argentina No 25.584 (2003), por su parte, establece que no podrá llevarse a cabo ningún tipo de acciones institucionales que impidan la normal prosecución de los estudios por parte de las estudiantes embarazadas o en período de lactancia. Se evita así que tengan lugar expulsiones o cambios compulsivos de escuela con base en decisiones arbitrarias de las autoridades escolares), tanto en establecimientos de gestión pública como privada.

\section{Oportunidades de reingresar al sistema: en búsqueda del éxito escolar en la adultez}

Los variados escenarios y oportunidades de reingresar al sistema educativo que las entrevistadas describieron tienen en común el hecho de haber logrado liberarse parcialmente, a lo largo de los tres años de duración del cursado, de sus responsabilidades domésticas. Para ello, contaron con la ayuda de otras mujeres pertenecientes a sus entornos familiares, que las reemplazaron en la realización de sus labores hogareñas y del cuidado de niños/as.

Una de las entrevistadas, Carmela (43 años), indica que sus hijas e hijo habían crecido y se encontraban ya en la escuela primaria cuando ella decidió volver a estudiar. Contaba con el apoyo de su madre y su hermana, con quienes vivía, para llevar adelante la casa y atender a los niños/as mientras ella estaba cursando. Además, disponía de ciertas condiciones económicas (su marido era policía y tenía ingresos estables) que le permitieron ir a estudiar en lugar de tener que salir a trabajar. En ese sentido, esta mujer y su grupo familiar podían afrontar el "costo de oportunidad" que la escolarización implicaba.

Por su parte, Anahí destaca que como tiempo de estudio disponía solo de las escasas horas de descanso que su trabajo a jornada completa le dejaba.

- Me habían dicho que esperara a cumplir los dieciocho y que me metiera a un CENS $^{6}$, porque yo estaba trabajando. Así que buenísimo, lo hice, porque eso no me interfería con mi trabajo, entonces seguía trabajando e iba a estudiar a la noche. (Anahí, 25 años. Entrevista 2 de marzo, 2007)

5 De acuerdo con la Ley № 24.195 (1993), conocida como Ley Federal de Educación y vigente en Argentina entre 1993 y 2006, la Educación Polimodal constituye un nivel de escolarización obligatoria, de tres años de duración. Es el nivel posterior a la Educación General Básica (de nueve años de duración, a partir de los 6 años de edad).

6 La sigla CENS corresponde a Centro Educativo de Nivel Secundario. 
URL: http://www.una.ac.cr/educare

Similares son los casos de otras entrevistadas, que aun sobrecargadas de actividades remuneradas y no remuneradas, decidieron cursar el nivel secundario a costa de sacrificar las reducidas horas libres que sus obligaciones les dejaban.

En la trayectoria de Luz, la imposibilidad de continuar realizando trabajos pesados que exigían un esfuerzo físico importante, como consecuencia de diversos problemas de salud, fue el factor detonante que le condujo a procurar alcanzar mejores niveles de preparación, con vistas a conseguir un trabajo menos rudo.

-Yo empecé el CENS debido a que yo trabajaba [...] en unos galpones de empaque de zapallo. O sea, era trabajo fuerte, trabajo pesado. Y a mí me hicieron tres operaciones seguidas. Me operaron una vez, primero, de una cesárea. Después me puse un DIU7, pasaron dos años, se me saltó el DIU, me tuvieron que operar. [...] Entonces me agarró pancreatitis y me tuvieron que volver a operar. ¿Entonces qué pasó? Ese trabajo que yo hacía, ya no lo podía seguir haciendo, porque era hacer fuerza, levantar cajas, levantar bultos y corría riesgo de herniarme.

Entonces mi familia me dijo: "Nosotros te vamos a ayudar para que vos estudiés. Porque nosotros te podemos bancar un mes, dos meses, tres meses, un año. Pero no te vamos a bancar toda la vida hasta que seas vieja." (Luz, 40 años. Entrevista 8 de abril, 2007)

Sumado a la cuestión de la sobrecarga de trabajo que para la mayoría de estas mujeres ha representado volver a estudiar, se encuentra la problemática económica. Al interior de la muestra, un grupo de mujeres muy pobres solo ha tenido la posibilidad de reingresar en el nivel medio debido a que resultaron beneficiarias de un programa social-educativo, el Plan Jefas de Hogar (PJH), inaugurado en Mendoza en el año 2000. A cambio de una prestación en dinero consistente en ciento cincuenta pesos mensuales, las beneficiarias debían completar el nivel secundario.

Diversas características de la organización de los establecimientos educativos para Jefas de Hogar pueden ser consideradas como "oportunidades" que coadyuvaron al éxito de la experiencia, que permitió que más de mil mujeres transitaran por el nivel medio en la provincia, entre 2000 y 2003, de acuerdo con datos del Ministerio de Desarrollo Social y Salud (2004). En primer lugar, el hecho de que se cursara en horarios diurnos -en turno mañana o tarde- fue un factor de fuerte peso inclusivo, ya que permitió que las mujeres fueran a cursar en el mismo horario en que sus hijos e hijas estaban en la escuela. La problemática de la violencia en la ciudad de Mendoza, que hace que mucha gente se sienta insegura al transitar por sus calles, conduce al aislamiento en el hogar de progresivamente mayor número de personas, entre ellas, las mujeres. Estas cuestiones contribuyeron a que las mujeres del PJH consideraran muy oportuna la posibilidad de ir a cursar en horario diurno.

La sigla DIU corresponde a Dispositivo Intrauterino. 
En cambio, quienes no pertenecían al PJH, como Luz, se veían compelidas a cursar en escuelas nocturnas.

- Me costó ir a la escuela, la gente podía opinar.

—¿Quién pensás que podía opinar?

—No sé... los vecinos, la gente amiga. "¡Mirá aquella!" Y era de noche, ¿viste? "¡Una mujer separada, cursando de noche!" [...] Entraba a las ocho y salía a las doce de la noche.

— ¿Te quedaba más o menos cerca, o muy lejos?

- Como un kilómetro y medio, por eso tenía que buscar a alguien con quien ir. Tenía terror. Un día que no fuera mi cuñado, ¿cómo hacía para volverme yo sola, a las doce de la noche, caminando? (Luz, 40 años. Entrevista 24 de julio, 2007)

El horario nocturno de cursado resulta, para muchas mujeres, poco atractivo o es percibido como inseguro. Inclusive, por razones que tienen que ver con un machismo arraigado en la sociedad, está mal visto que las mujeres anden solas por la calle en ese horario ("Mirá aquella"; "Una mujer separada cursando de noche").

Por todas estas razones, y volviendo al PJH, el cursado diurno fue muy bien recibido por las mujeres involucradas en ese programa social.

En segundo lugar y respecto de la infraestructura, se abrieron nuevos establecimientos para adultas, pero no se construyeron nuevas escuelas. En cambio, se recurrió a las más variadas estrategias para subsanar el problema edilicio, desde el aprovechamiento de edificios o aulas abandonados que debieron ser reciclados, hasta la realización de convenios con organizaciones comunitarias e instituciones religiosas barriales, que autorizaron el uso de sus espacios físicos a los fines educativos. Inclusive, fueron alquilados algunos salones y viviendas que podían ser utilizados como aulas. Buena parte de la infraestructura que se montó resultaba poco apropiada para los requerimientos educativos, pues los espacios eran pequeños, carecían del mobiliario necesario o no disponían de instalaciones básicas como sanitarios suficientes, cocinas o patios de recreos. "(...) Son generalmente las escuelas que atienden a sectores populares las que cuentan con peores características edilicias, de equipamiento, de capacitación de sus docentes y de tiempo de aprendizaje efectivo" (Gluz et al., 2002, p. 36).

Sin embargo, el hecho de que dieciséis nuevas escuelas de nivel medio pudieran montarse en menos de seis meses sin que para ello se construyera ningún nuevo edificio escolar, habla a las claras de que cuando hay voluntad política de hacer frente a necesidades educativas consideradas urgentes y críticas, ciertas transformaciones pueden implementarse. 
URL: http://www.una.ac.cr/educare

Los establecimientos se instalaron en las cercanías de los lugares de residencia de las estudiantes, lo cual constituye otro factor de inclusión educativa. Se intentó así evitar que las mujeres tuvieran que invertir demasiado tiempo y dinero en trasladarse hasta la escuela. La mayoría hacía el trayecto en bicicleta o caminando, incluso cuando las distancias por recorrer eran de varios kilómetros.

Por último, el aspecto quizás más destacado fue la creación de Unidades de Cuidado Infantil (UCI) para los hijos e hijas en edad preescolar de las alumnas. Sin dudas, muchas de ellas no hubieran logrado terminar sus estudios de no haber contado con un espacio seguro y gratuito para dejar a sus hijos/as en las horas de clases. Además, la cercanía de las UCl y las aulas de las mujeres permitía un cierto contacto que facilitaba actividades como la lactancia materna.

En definitiva, el conjunto de dispositivos y servicios inaugurados por el PJH debe ser concebido como la oportunidad que condujo a que las mujeres pudieran reingresar al sistema educativo y permanecer en él hasta completar el nivel secundario. De hecho, muchas de las entrevistadas consideraban que no estaba en su proyecto vital volver a estudiar antes de la creación del Plan Jefas de Hogar. Este abrió para ellas un nuevo escenario, en el que la conclusión del nivel medio se tornó en una aspiración legítima y sostenible a lo largo de los tres años de duración del cursado.

\section{Algunas conclusiones}

El análisis de la díada fracaso/éxito escolar ha permitido poner de manifiesto el carácter social de los procesos que configuran las trayectorias educativas de las mujeres de sectores populares en Mendoza. Así, puede afirmarse que el fenómeno del fracaso escolar no debe tener como responsables a aquellas que se educan, sino a la propia institución educativa. La que fracasa, desde este enfoque, es la escuela, como parte de un orden social profundamente injusto. Pero cambiar la perspectiva y afirmar, como lo hacemos aquí, que la responsable de esta problemática es una institución y no las personas escolarizadas no libera a estas últimas de las consecuencias del fracaso escolar.

En América Latina, las mujeres deben completar en promedio cuatro años de escolarización formal más que los varones para obtener el mismo ingreso que ellos, en una ocupación similar (Aguirre, 2005). Así, son ellas las que sufren en carne propia las consecuencias del abandono escolar, que las afecta diferencialmente por su condición de género. Esto pudo verificarse en el trabajo de campo, puesto que quienes abandonaron la escuela en su infancia o adolescencia han estado indefectiblemente destinadas a realizar las labores peor posicionadas y remuneradas en toda la estructura ocupacional.

Más aún, las consecuencias del fracaso escolar se extienden para toda la vida, ya que incluso cuando estas mujeres pudieron completar el nivel medio en la adultez, sus posibilidades 
de acceso a puestos de trabajo calificados no se han modificado significativamente, y la mayoría de ellas continuaba inserta en empleos precarios, al momento de la finalización de nuestro trabajo de campo en 2007.

Respecto de las "oportunidades" que este grupo de mujeres aprovechó para volver a estudiar, debemos destacar que distan de constituir escenarios claramente favorables en todos los casos. Debieron llevar a cabo verdaderas "reingenierías" del tiempo y las responsabilidades domésticas, lo que les demandó importantes esfuerzos y sacrificios en términos personales, requirieron además del apoyo de otras mujeres de sus grupos convivientes. De no haber contado con esa colaboración -que no es otra cosa que el aporte del trabajo doméstico no remunerado de sus madres, hijas o hermanas, y que descansa sobre una división sexual del trabajo y las responsabilidades muy injusta para las mujeres- no hubiesen podido, según ellas mismas han señalado, concluir con éxito la escolarización en la adultez.

En relación con el grupo de estudiantes que asistieron a establecimientos creados por el $\mathrm{PJH}$, hemos mencionado que antes de ingresar, estas mujeres no consideraban que estuvieran en condiciones de continuar estudiando $y$, por ello, volver a la escuela no formaba parte de sus proyectos vitales. La inauguración de escuelas de adultas que efectivamente han tenido en cuenta las condiciones de existencia de sus poblaciones estudiantiles (con turnos de cursado diurno, un programa social que aseguraba un ingreso mensual fijo para las estudiantes y la creación de unidades de cuidado infantil, entre otros factores) muestra que quienes antes había sido excluidas y consideradas poco inteligentes o poco predispuestas para la actividad académica, ahora -ya adultas- han sido capaces de protagonizar trayectorias escolares "exitosas".

En definitiva, es la modificación de las condiciones institucionales y no un cambio en las disposiciones o capacidades individuales lo que ha permitido que este grupo de personas alcance la terminalidad educativa.

Hemos indicado que las escuelas para personas adultas forman parte de un circuito escolar diferencial, y esto también produce efectos sobre las oportunidades de ver mejoradas las propias condiciones de vida de sus estudiantes. Atender a poblaciones cuyos capitales culturales son diferentes al capital escolarmente rentable (el cual coincide, a su vez, con el capital cultural de los grupos sociales más favorecidos), así como el hecho de que los trayectos educativos tengan una duración significativamente menor, son factores que coadyuvan a que la calidad de la educación recibida sea más baja. Por ende, las capitalizaciones posibles en las escuelas de adultos, muchas veces, resultan insuficientes para el tipo de exigencias que el mercado de trabajo plantea, o para enfrentar, de manera exitosa, la continuidad de los estudios en el nivel superior.

No obstante, el trabajo de campo permite afirmar que los CENS en Mendoza, aún constituyendo un circuito educativo diferencial de baja calidad, dan lugar a procesos de apropiación de conocimientos y experiencias que los sujetos escolarizados consideran necesarios y muy valiosos. 
URL: http://www.una.ac.cr/educare

\section{Referencias}

Agencia informativa católica argentina [AICA]. (27 de agosto de 2008). Champagnat, una escuelita en los contrafuertes andinos. AICA On Line. Recuperado de http://aica.org/index. php?module $=$ displaystory \&story id=13453\&format $=$ html

Aguirre, R. (2005). Apuntes para el Seminario Virtual: Pobreza, globalización y género. Avances teóricos, de investigación y estrategias en América Latina. Manuscrito inédito, Programa Regional en Género y Políticas Públicas (PRIGEPP), FLACSO. Buenos Aires.

Bourdieu, P. (2000). Comprender. En P. Bourdieu (Dir.). La miseria del mundo (pp. 527-556). Buenos Aires: Fondo de Cultura Económica.

Bourdieu, P. (2006). La distinción. criterio y bases sociales del gusto (3a ed.). Madrid: Taurus.

Cortese, C., Romagnoli, M. C., Bustos, R. M. y Castillo, M. N. (1999). El fracaso escolar: ¿Tema antiguo o problema candente? Mendoza: Ediunc.

Equipo Latinoamericano de Justicia y Género [ELA]. (2005). Informe sobre género y derechos humanos. Vigencia y respeto de los derechos de las mujeres en Argentina. Buenos Aires: Biblos.

Fainsod, P. Y. (2006). Embarazo y maternidad adolescente en la escuela media. Una discusión sobre las miradas deterministas de las trayectorias escolares de adolescentes embarazadas y madres en contextos de pobreza. Buenos Aires: Miño y Dávila.

Furbank, P. N. (2005). Un placer inconfesable o la idea de clase social. Buenos Aires: Paidós.

García-Huidobro, J. E. (1994). Los cambios en las concepciones actuales de la educación de adultos. En UNESCO/UNICEF (ed.). La educación de adultos en América Latina ante el próximo siglo (pp. 15-50). Santiago, Chile: OREALC/UNESCO/UNICEF.

Gardner, H., Kornhaber, M. L. y Wake, W. K. (2005). Las perspectivas biológicas. En Inteligencia: Múltiples perspectivas (95-124). Buenos Aires: Aique.

Gluz, N., Kantarovich, G. y Kaplan, C. (2002). La autoestima que fabrica la escuela. En C. Kaplan (Comp.). La escuela: Una segunda oportunidad frente a la exclusión. El deseo de aprender: Un antídoto al fracaso escolar. De la discriminación oculta a la democratización en la escuela. (pp. 15-46). Argentina: Novedades educativas.

Instituto Nacional de Estadísticas y Censos [INDEC]. (2001). Censo nacional de población, hogares y vivienda 2001. Recuperado de http://www.indec.gov.ar/censo2001s2/ampliada index. asp?mode $=01$

Instituto Nacional de Estadísticas y Censos [INDEC]. (2006). Incidencia de la pobreza y de la indigencia en 28 aglomerados urbanos. Resultados $1^{\circ}$ semestre de 2006. Recuperado de: http://www.indec.gov.ar/nuevaweb/cuadros/74/pob tot 1sem06.pdf 
Ley No 24.195. (1993). Ley Federal de Educación. República Argentina.

Ley No 25.273. (2000). Régimen de inasistencias justificadas por razones de gravidez. República Argentina.

Ley No 25.584. (2003). Inicio o continuidad del ciclo escolar de estudiantes embarazadas o en período de lactancia. República Argentina.

Ministerio de Desarrollo Social y Salud. (2004). Plan Jefas de Hogar. Programa social educativo. Mendoza: Gobierno de Mendoza.

Moser, C. (1996). Situaciones críticas. Reacción de los hogares de cuatro comunidades urbanas pobres ante la vulnerabilidad y la pobreza. (Serie de estudios y monografías sobre el desarrollo ecológicamente sostenible, 75). Washington: Banco Mundial.

Romagnoli, M. C., Maure, G. E., Cortese, C., Draghi, M. J., Sarmiento, C., Tosoni, M. y Pérez, A. (1998). Los ejes de inclusión-exclusión en el sistema educativo: Una propuesta alternativa. En M. V. Gómez (Comp.). A propósito de los viejos y nuevos problemas educativos (pp. 157172). Mendoza: Facultad de Educación Elemental y Especial.

Scott, J. W. (1990). El género. Una categoría útil para el análisis histórico. En J. S. Amelang y M. J. Nash (Eds.). Historia y género: Las mujeres en la Europa moderna y contemporánea (pp. 23-56). España: Universidad de Valencia.

Tenti, E. (2007). La escuela y la cuestión social. Ensayos de sociología de la educación. Buenos Aires: Siglo XXI. 